\title{
ON THE SCALING OF BUBBLE CLUSTER COLLAPSE IN CLOUD CAVITATING FLOW AROUND A SLENDER PROJECTILE
}

\author{
Yiwei Wang \\ Key Laboratory for Mechanics in \\ Fluid Solid Coupling Systems, \\ Institute of Mechanics, Chinese \\ Academy of Sciences \\ Beijing, China
}

\author{
Chenguang Huang \\ Key Laboratory for Mechanics in \\ Fluid Solid Coupling Systems, \\ Institute of Mechanics, Chinese \\ Academy of Sciences \\ Beijing, China
}

\author{
Xiaocui Wu \\ Key Laboratory for Mechanics in \\ Fluid Solid Coupling Systems, \\ Institute of Mechanics, Chinese \\ Academy of Sciences \\ Beijing, China
}

\begin{abstract}
The scaling law of bubble cluster collapse in cloud cavitating flow around a slender projectile is investigated in the present paper. The influence of compressibility is mainly discussed. Firstly the governing parameters are obtained by dimensional analysis, and the numerical method is established in order to verify the similarity law and obtain the influence of parameters based on a mixture approach with Singhal cavitation model. Moreover, the similarity law is validated by numerical simulations. Two main factors of compressibility of mixture fluid, including compressibility of non-condensable gas and phase change, are studied, respectively. Results indicated that the phase change has little influence on both flowing and collapse pressure. In the condition that the variation range of the mixture compressibility is small, the compressibility of non-condensable gas has notable impact the local collapse pressure peaks, however the macroscopic flow pattern does not change.
\end{abstract}

\section{INTRODUCTION}

When cavitation bubbles collapse, high pressure pulses were generated, which have major impact on vehicles structure and may even cause damage [1]. The collapse of bubble cluster is also an important issue especially for engineering applications.

Classic research on cavitation collapse usually focuses on collapse of single bubble and finite number of bubbles. Theoretical analysis can be performed on the basis of RayleighPlesset equation $[2,3]$, to obtain the characteristics of single bubble collapse, and interactions between limited number of bubbles are also studied [5-8].

For the cloud cavitating flow, instabilities at cavitation closures are usually prominent, with quasi-periodical breaking- off and shedding [9, 10]. It was observed that shedding cavitation cloud may collapse at the cavitation closure, which is also closely related to the instability of cavitation bubbles [1114]. The usual governing dimensionless parameters of similarity law are cavitation number and Reynolds number. However, impact effect is significant in collapse process, which is relative to compressibility [15]. The scaling law of bubble colluster collapse is worth discussing including the compressible.

In the present paper, typical cavitating flow and bubble cluster collapse phenomena around a slender projectile are investigated. The governing parameters are obtained by dimensional analysis. The similarity law is validated by numerical simulations. Two main factors of compressibility of mixture fluid, including compressibility of non-condensable gas and phase change, are studied, respectively. The influences on the macroscopic flow and local collapse pressure are discussed.

\section{THEORETICAL ANALYSIS}

In the bubble collapse process, the mixture fluid impacts intensely on the wall surface, inducing pressure pulse with high peak. Pressure peak of flow impact can be calculated as $p_{i}=\rho_{m} v_{i} c_{m}$, where $p_{i}$ is the impact pressure, $v_{i}$ is the impact velocity, $\rho_{m}$ and $c_{m}$ are the density and sound speed of mixture, respectively. Sound speed is the most important parameter to describe the compressibility of mixture. The sound speed of water containing air is represent as 


$$
\begin{aligned}
& c_{m}=\left(\frac{1}{\rho_{m} \mathrm{~K}_{m}}\right)^{\frac{1}{2}} \\
& =\left\{\frac{1}{\left[\alpha_{a} \rho_{a}+\left(1-\alpha_{a}\right) \rho_{w}\right] \cdot\left[\alpha_{a} \mathrm{~K}_{a}+\left(1-\alpha_{a}\right) \mathrm{K}_{w}\right]}\right\}^{\frac{1}{2}}
\end{aligned}
$$

where $\mathrm{K}_{m}, \mathrm{~K}_{a}$ and $\mathrm{K}_{w}$ are compression modulus of mixture, air and water, respectively. $\rho_{a}$ and $\rho_{w}$ are the densities of air and water, respectively. $\alpha_{a}$ is the volume fraction of air.

Considering $\mathrm{K}_{w} \gg \mathrm{K}_{a}$, the compressibility of pure water can be neglected. The formula of sound speed is simplified as following,

$$
c_{m}=\frac{c_{a}}{\alpha_{a}}\left(\frac{1}{1+\frac{1-\alpha_{a}}{\alpha_{a}} \cdot \frac{\rho_{\mathrm{w}}}{\rho_{a}}}\right)^{\frac{1}{2}}
$$

where $c_{a}=\left(\frac{1}{\rho_{a} \mathrm{~K}_{a}}\right)^{\frac{1}{2}}$ is the sound speed of air. Because the volume fractions are related to the flow evolution, the sound speed of air is the main parameter of material properties to represent the compressibility of mixture.

Moreover, the governing parameters of typical cloud cavitating flow are listed as

$$
p=f\left(\rho_{w}, \rho_{a}, v, D, \mu, g, p_{\infty}-p_{v}, T_{s}, N, c_{a}, t\right)
$$

where $p$ is the pressure, $v$ is speed of the projectile motion, $D$ is the diameter, $\mu$ and $T_{s}$ are the laminar viscosity coefficient and the coefficient of surface tension of water, respectively, $g$ is the acceleration of gravity, $p_{\infty}-p_{v}$ represents the difference of background pressure and saturation pressure, $N$ is the number density of non-condensable bubble nuclei, and $t$ is the time. The formula is nondimensionalized as,

$$
\frac{p}{\frac{1}{2} \rho_{w} v^{2}}=f\left(\frac{\rho_{w}}{\rho_{a}}, \operatorname{Re}, \mathrm{Fr}, \sigma, \mathrm{We}, N^{1 / 3} D, \mathrm{Ma}, \frac{t v}{D}\right)
$$

where, $\operatorname{Re}=\frac{\rho_{w} v D}{\mu}$ is the Reynolds number, $\sigma=\frac{p_{\infty}-p_{v}}{\frac{1}{2} \rho_{w} v^{2}}$ is the cavitation number, $\mathrm{We}=\frac{\rho_{w} v^{2} D}{T_{s}}$ is the Weber number, $\mathrm{Ma}=\frac{v}{C_{a}}$ is the Mach number, and $\frac{t v}{D}$ is the dimensionless time. This formula contains the main parameters and constitutes the similarity law considering compressibility effects of the flowing and collapse process.

\section{NUMERICAL METHOD}

In order to verify the similarity law and obtain the influence of compressibility, numerical simulations are carried out. The numerical method was based on the mixture approach with Singhal cavitation model and modified RNG k- $\varepsilon$ turbulence model, and the non-condensable gas is also considered as the original Singhal model [16]. The continuity and momentum equations for the mixture of liquid water, vapor, and non-condensable air are established as

$$
\begin{aligned}
& \frac{\partial}{\partial t}\left(\rho_{m}\right)+\nabla \bullet\left(\rho_{m} \vec{v}_{m}\right)=0 \\
& \frac{\partial}{\partial t}\left(\rho_{m} \vec{v}_{m}\right)+\nabla \bullet\left(\rho_{m} \vec{v}_{m} \vec{v}_{m}\right) \\
& =-\nabla p+\nabla \bullet\left[\left(\mu_{m}+\mu_{t}\right)\left(\nabla \vec{v}_{m}+\nabla \vec{v}_{m}^{\mathrm{T}}\right)\right]
\end{aligned}
$$

where $\vec{v}_{m}$ is the mixture velocity vector. The laminar viscosity $\mu_{m}$ is defined as a density-weighted average of the three components. $\mu_{t}$ is the turbulent viscosity closed by a modified RNG k- $\varepsilon$ model[17]. The mixture density $\rho_{m}$ is defined by

$$
\frac{1}{\rho_{m}}=\frac{f_{v}}{\rho_{v}}+\frac{f_{a}}{\rho_{a}}+\frac{1-f_{v}-f_{a}}{\rho_{w}}
$$

where $f_{v}$ and $f_{a}$ are the component mass fractions. $\rho_{v}$ is the density of the vapor. During calculation, $f_{a}$ is assumed to be a small constant as $15 \mathrm{ppm}$.

The mass fraction equation for vapor is

$$
\frac{\partial}{\partial t}\left(\rho_{m} f_{v}\right)+\nabla\left(\rho_{m} \overrightarrow{v_{m}} f_{v}\right)=R_{e}-R_{c}
$$

where $R_{e}$ and $R_{c}$ are the evaporation and condensation rates, respectively, which can be simulated in the cavitation model established by Singhal [16] .

$$
\left\{\begin{array}{l}
R_{e}=C_{e} \frac{\sqrt{k}}{T_{s}} \rho_{w} \rho_{v} \sqrt{\frac{2\left(p_{v}-p\right)}{3 \rho_{w}}}\left(1-f_{v}-f_{a}\right), p<p_{v} \\
R_{c}=C_{c} \frac{\sqrt{k}}{T_{s}} \rho_{w} \rho_{w} \sqrt{\frac{2\left(p-p_{v}\right)}{3 \rho_{w}}} f_{v}, p>p_{v}
\end{array}\right.
$$

In this model, $k$ is the turbulence kinetic energy. $C_{e}$ and $C_{c}$ are two empirical coefficients have dimension as the same as velocity, and are set as 0.02 and 0.01 in the following simulation by default, respectively.

The phase change phenomenon reflects the relationship between pressure and density, which is also essentially a manifestation of compressibility. Therefore, on the basis of Eq.(4), the expression of dimensionless parameters including the phase change effect is further derived as following.

$$
\frac{p}{\frac{1}{2} \rho_{w} v^{2}}=f\left(\frac{\rho_{w}}{\rho_{a}}, \mathrm{Re}, \mathrm{Fr}, \sigma, \mathrm{We}, N^{1 / 3} D, \mathrm{Ma}, \frac{C_{e}}{v}, \frac{C_{c}}{v}\right)
$$

These unsteady numerical simulations were performed based on the finite volume method with the SIMPLEC scheme by using the commercial CFD software FLUENT. The 
equations are discretized by a second order implicit scheme in time and a second order upwind scheme in space. The computational domain was discretized with a block structured grid. The height of the first layer was set as $1 / 10000$ of the projectile's diameter to ensure the $y+$ equals to 1 approximately. Thus the two-layer model was adopted to resolve the laminar sub layer. For the velocity inlet boundary condition in front of the projectile, the inlet velocity is set as $17.3 \mathrm{~m} / \mathrm{s}$, and the turbulent intensity is set as $1 \%$ and the hydraulic diameter is set as $0.037 \mathrm{~m}$. Therefore the inlet $\mathrm{k}$ and $\varepsilon$ can be calculated as 0.045 and 0.56 . For the pressure outlet boundary condition downstream of the projectile, the pressure is set as $1 \mathrm{~atm}$.

Details about the computational configurations can be found in references [18] and [19], and was validated by comparing with a typical experiment result of cavitating flow around a slender projectile. The length and thickness obtained by numerical simulation agree well with the experimental results (as shown in Fig.1, the deviation of experimental observation is about $0.5 \mathrm{~mm}$ obtained by pixel analysis, which is also $1.35 \%$ of the diameter).

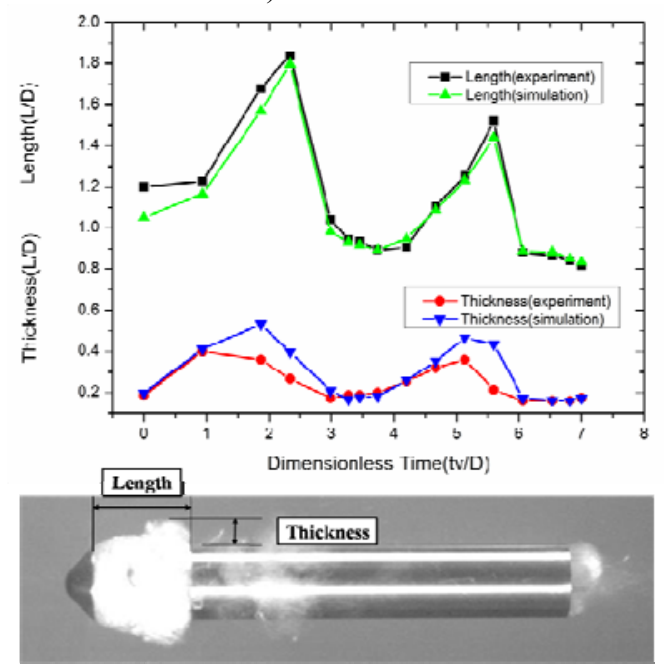

Fig.1 Validation of numerical simulation and a typical photograph

\section{RESULTS AND DISCUSSION}

\subsection{Cavities evolution}

The unsteady cloud cavitating flow around a slender cylinder with a conical head is investigated. The diameter of the projectile is $37 \mathrm{~mm}$, and the velocity is $17.3 \mathrm{~m} / \mathrm{s}$. Thus the cavitation number can be calculated out as 0.66 .

The cavitation bubbles grow, break up, shed and collapse periodically. When the re-entry jet just reaches the shoulder, cavitation bubbles collapse at the shoulder point (as shown in Fig.2-A1). New cavities grow and remain cavities shed (as shown in Fig.2 A2-A3). The shedding cavities shrink finally, meanwhile new re-entry jet forms (as shown in Fig. 2- A4). Reentry jet moves upstream and crosses with the main flow (as shown in Fig.2 A5-1 and A5-2). Re-entry jet reaches the shoulder, upstream part of the cavities collapse at the shoulder point. This time is also equivalent to the 1 st status of the next cycle (as shown in Fig.2-A6 also numbered as B1).

The most obvious collapse phenomenon occurs at the shoulder point on projectile surface (as shown in Fig. 2 A1 and B1). The re-entry jet flows to the upstream flow and compresses the cavity. The cavity finally collapses and high pressure is generated, which induces the remaining cavity to shed in the following.

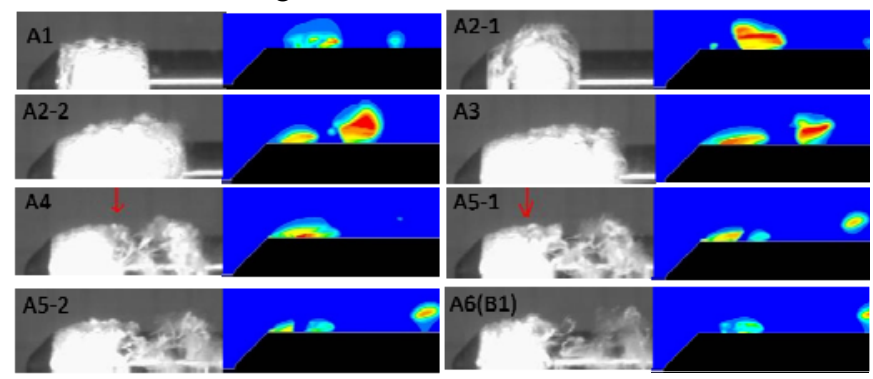

Fig.2 Cavities evolution in experimental and numerical results

\subsection{Validation of the similarity law}

In order to study the impact parameters quantitatively, pressure variations at several probes on the surface are measured and compared with different scales and parameter settings. One probe is set at the shoulder point, which is used to represent the local pressure variation of collapse. The other probe is set on diameter distance away from the shoulder and on the surface, in order to measure the pressure fluctuation at cavity closure. In addition to the original case, the scale of projectile is enlarged four times, and the velocity is enlarged two times. The parameters of material properties are modified appropriately to maintain all dimensionless parameters on the right side of Eq.(10). The variations of dimensionless pressure coefficients $C p=\frac{p}{\frac{1}{2} \rho_{w} v^{2}}$ are compared. The flowing pressure fluctuations at the cavity closure in numerical results are shown in Fig.3. With the growth and shedding, the pressure increases and decreases alternatively.

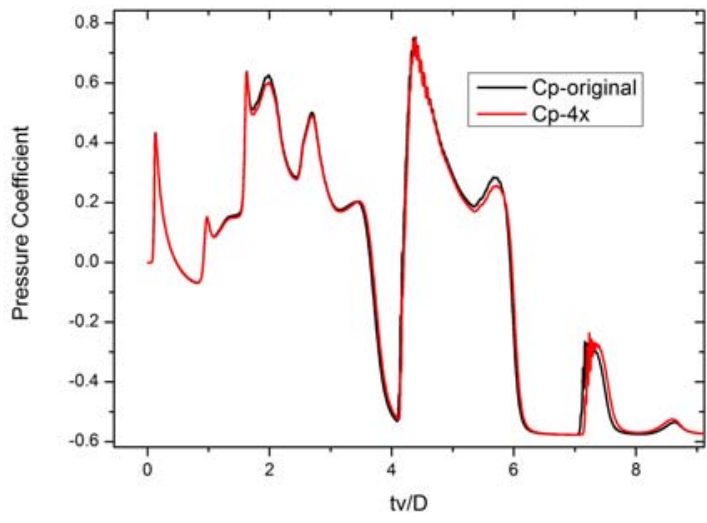

Fig. 3 Comparison of flowing pressure with different scales (probe at $\mathrm{x} / \mathrm{D}=1.5$ ) 
Collapse pressure peaks at the shoulder points are shown in Fig.4. The peak value is about four times of the stagnation pressure.

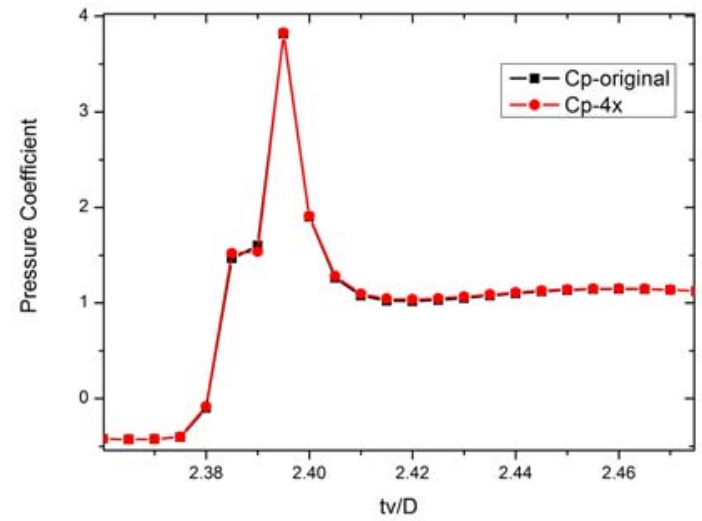

Fig.4 Comparison of collapse pressure with different scales (probe at $\mathrm{x} / \mathrm{D}=0.5$ )

As seen from the aforementioned two figures, the pressure curves agree well with each other between the original and enlarged scale cases. The similarity law with all parameters is validated.

\subsection{Influence of phase change}

On the basis of similarity with all parameters, the influences of compressibility on the flowing and collapse pressure are mainly discussed. The phase change rate is enlarged 10 times by increase the parameters of $C_{e}$ and $C_{c}$, while other dimensionless parameters are maintained. The pressure variations at the two probes are compared. The flowing pressure fluctuations at the cavity closure are shown in Fig.5. Inside this change range, the phase change rate has almost no impact on the flowing pressure. We can refer to the analysis in reference [20]. For the cloud cavitating flow, the phase change effect is much smaller than the convection of liquid phase, and the characteristic time of phase change is also much smaller than that of macroscopic flow. Therefore the influence of phase change rate can be neglected.

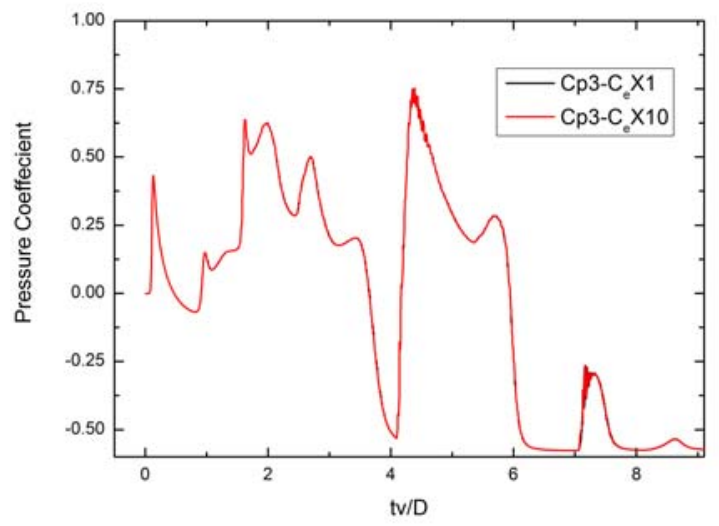

Fig.5 Comparison of flowing pressure with different phase change rates (probe at $\mathrm{x} / \mathrm{D}=1.5$ )

The comparison of local peaks of collapse pressure is shown in Fig. 6. The phase change rate has also little effect on the collapse pressure. This may because the collapse pressure is generated when the cavity shrinks to a very small volume, and at this time the phase change process almost finishes. The residual non-condensable air may be the main influential factor.

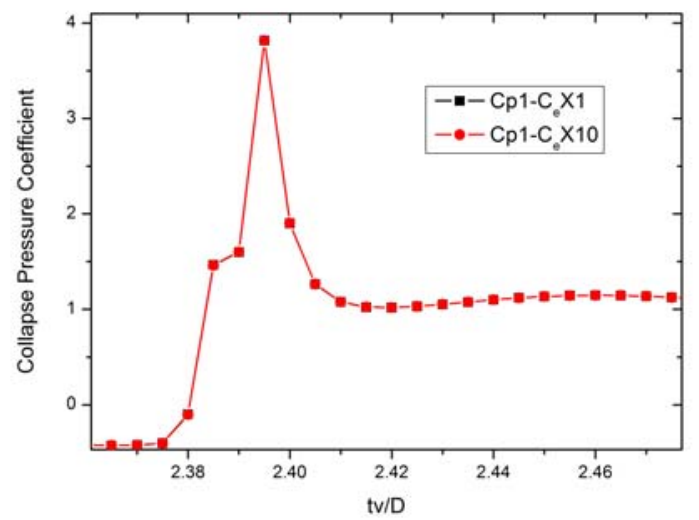

Fig.6 Comparison of collapse pressure with different phase change rates (probe at $\mathrm{x} / \mathrm{D}=0.5$ )

\subsection{Influence of the sound speed of air}

On the basis of similarity with all parameters, the sound speed of non-condensable are is enlarged twice and 10 times, while other dimensionless parameters are maintained. The pressure variations at the two probes are compared. As shown in Fig. 7, the overall shapes of collapse pressure peaks are similar. The pressure peaks in the high sound speed conditions are notably higher than the original condition, but the increasing magnitude of pressure is less than the speed of sound. This shows that the impact pressure $p_{c}=\rho_{m} v_{c} c_{m}$ and the flow stagnation pressure both occupy certain proportions in the collapse pressure. In case of changing the speed, the former corresponding changes, while the latter is almost unchanged.

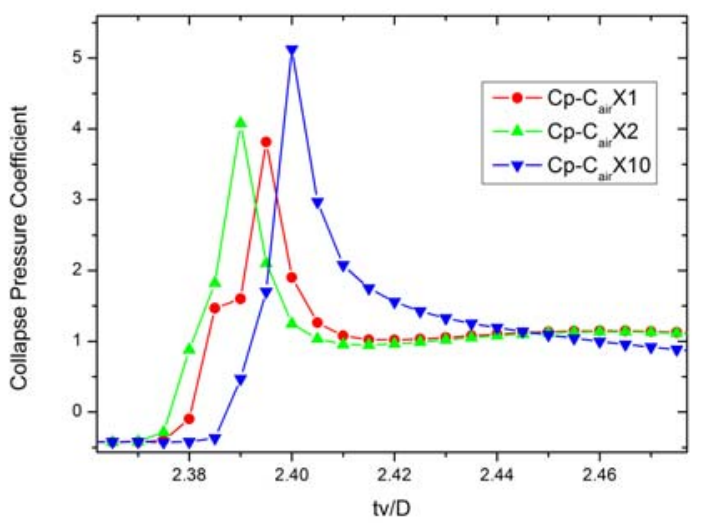

Fig.7 Comparison of collapse pressure with different sound speeds of air (probe at $\mathrm{x} / \mathrm{D}=0.5$ )

The flowing pressure fluctuations at the cavity closure are compared between the original and changing sound speed conditions as shown in Fig.8. Inside this change range, the sound speed has little effect on the flowing pressure. Therefore, the impact effect is limited in the local area and time period of 
collapse, and the overall impulse is also small. It has almost no impact on the macroscopic flowing pressure.

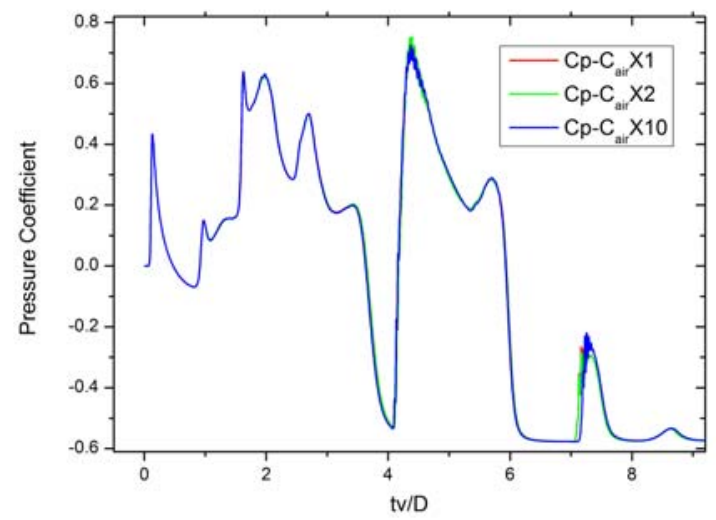

Fig.8 Comparison of flowing pressure with

\section{CONCLUSIONS}

different sound speeds of air (probe at $\mathrm{x} / \mathrm{D}=1.5$ )

The governing parameters of cloud cavitating flow considering compressibility effects on collapse, including cavitation number, Reynolds number, Mach number and dimensionless phase change rate, are derived. Different scale cases are simulated. Pressure variations at several probes are compared to estimate the influence of derived dimensionless parameters. Results indicate that:

1. When all the parameters are maintained in different scale cases, the results are exactly similar.

2. The phase change has little effect on both collapse pressure and flowing pressure when it varies in a not very large range. This is because the phase change occurs mainly in the macroscopic flow process, which is dominated by the motion of liquid phase.

3. The compressibility of non-condensable gas has notable impact on the collapse pressure. In the condition that the variation range of the mixture compressibility is small, the Mach number only affects the local collapse pressure peaks, and the macroscopic flow pattern does not change.

The issues of governing parameters and scaling law of cavitation collapse pressure are very complicated but also of great importance for the engineering applications. We attempt to derive the source of scale effects from a theoretical perspective in the present paper, and validate by numerical methods. Our work is still preliminary, and we hope it will serve as a modest spur to induce someone to come forward wit $\mathrm{h}$ his valuable contributions, and will be verified and validated by rigorous experimental results in the future.

\section{ACKNOWLEDGMENTS}

This research was sponsored by National Natural Science Foundation of China (Grant numbers 11332011 \& 11202215) and the Youth Innovation Promotion Association of CAS (Grant number 2015015).

\section{REFERENCES}

[1] Brennen, C. E. Cavitation and bubble dynamics. Oxford University Press, USA, 1995.

[2] Plesset, M. S., and Chapman, R. B., 1971, "Collapse of an initially spherical vapour cavity in the neighborhood of a solid boundary," Journal of Fluid Mechanics, 47(2), pp.283-290. [3] Plesset, M. S., Prosperetti, A. Bubble dynamics and cavitation. Annual Review of Fluid Mechanics, 1977, 9(1): 145-185.

[4] Seo, J. H., Lele, S. K., and Tryggvason, G., 2010, "Investigation and modeling of bubble-bubble interaction effect in homogeneous bubbly flows," Physics of Fluids, 22(6), p.063302.

[5] Bremond, N., Arora, M., Ohl, C.-D., and Lohse, D., 2006, "Controlled multibubble surface cavitation," Physical Review Letters, 96(22), p.224501.

[6] Lauer, E., Hu, X. Y., Hickel, S., and Adams, N. A., 2002, "Numerical investigation of collapsing cavity arrays," Physics of Fluids, 24(5), p.052104.

[7] Pelekasis, N. A., Gaki, A., Doinikov, A., and Tsamopoulos, J. A., 2004, "Secondary bjerknes forces between two bubbles and the phenomenon of acoustic streamers," Journal of Fluid Mechanics, 500, pp. 313-347.

[8] Quinto-Su, P. A., and Ohl, C. D., 2009, " Interaction between two laser-induced cavitation bubbles in a quasi-twodimensional geometry," Journal of Fluid Mechanics, 633, pp.425-435.

[9] Callenaere, M., Franc, J. P., Michel, J. M., and Riondet, M, 2001, "The Cavitation Instability Induced by the Development of a Re-Entrant Jet," Journal of Fluid Mechanics, 444, pp.223256.

[10] Le, Q., Franc, J., and Michel, J, 1993, "Partial Cavities: Pressure Pulse Distribution around Cavity Closure," Journal of Fluids Engineering, 115(2), pp.249-254.

[11] Reisman, G. E., Wang, Y., and Brennen, C. E., 1998, "Observations of shock waves in cloud cavitation," Journal of Fluid Mechanics, 355(1), pp.255-283.

[12] Saito, Y., Takami, R., Nakamori, I., and Ikohagi T., 2007, "Numerical analysis of unsteady behavior of cloud cavitation around a naca0015 foil," Computational Mechanics, 40(1),pp. 85-96.

[13] Konno, A., Kato, H., Yamaguchi, H., and Maeda, M., 2002, "On the collapsing behavior of cavitation bubble clusters," JSME International Journal Series B, 45(3), pp.631-637. [14] Saito, Y., and Sato, K., 2003, "Cavitation bubble collapse and impact in the wake of a circular cylinder," Fifth International Symposium on Cavitation (CAV2003), Osaka, Japan.

[15] Wang, Y., Liao, L., Du, T., et al, 2014, "A study on the collapse of cavitation bubbles surrounding the underwaterlaunched projectile and its fluid-structure coupling effects," Ocean Engineering, 84, pp. 228-236.

[16] Singhal, A. K., Athavale, M. M., Li, H., and Jiang, Y., 2002, "Mathematical Basis and Validation of the Full Cavitation Model," ASME J. Fluids Eng., 124(3), pp. 617-624. 
[17] Dular, M., Bachert, R., Stoffel, B., and Sirok, B., 2005, "Experimental evaluation of numerical simulation of cavitating flow around hydrofoil," European Journal of Mechanics B/Fluids, 24(4), pp. 522-538.

[18] Wei, Y. P., Wang, Y. W., Fang, X., Huang, C. G., and Duan, Z. P., 2011, "A Scaled Underwater Launch System Accomplished by Stress Wave Propagation Technique," Chinese Physics Letters, 28, p. 024601.
[19] Wang, Y., Huang, C., Fang, X., Du, T., and Yu, X., 2013, "Characteristics of the re-entry jet in the cloud cavitating flow over a submerged axisymmetric projectile (in Chinese)," Chinese Journal of Hydrodynamics, 28(1), pp. 23-29.

[20] Yu, X., Wang, Y., Huang, C., and Du, T., 2013, "Study on the influence of phase change rate on cloud cavitation,"

Procedia Engineering, 61, pp.204-206. 